\title{
Produtividade de frutos e nutrição mineral de pepineiro para conserva submetido a soluções nutritivas em semi-hidroponia
}

\author{
João Vieira Neto' ${ }^{1}$ Rafael Gustavo Ferreira Morales² e Cristiano Mora ${ }^{3}$
}

\begin{abstract}
Resumo - O presente trabalho foi realizado com objetivo de avaliar concentrações da solução nutritiva sobre a produtividade e qualidade de frutos de pepineiro para conserva, sob ambiente protegido. Dois experimentos foram conduzidos na Estação Experimental de Ituporanga (Epagri/EEltu), em SC, nos anos de 2019 e 2020, com o híbrido Kybria F1. O delineamento experimental foi o de blocos casualizados com seis repetições, sendo os tratamentos: 80, 90, 100, 110 e $120 \%$ das quantidades de fertilizantes recomendadas pela pesquisa, seguindo tabela de distribuição indicada pela indústria para cada estágio de desenvolvimento. Houve efeito significativo das concentrações de soluções nutritivas avaliadas sobre a produtividade do pepineiro. Os tratamentos que proporcionaram maior desempenho produtivo, utilizando o manejo indicado pela indústria, foram $90 \%$ da solução padrão e solução padrão $100 \%$.
\end{abstract}

Termos de indexação: Cucumis sativus; fertirrigação; fases fenológicas.

\section{Fruit yield and mineral nutrition of pickling cucumber subjected to nutrient solutions in semi-hydroponics}

Abstract - The present work was carried out with the objective of evaluating concentrations of the nutrient solution on the productivity and quality of pickling cucumber fruits under protected cultivation. Two experiments were conducted at Estação Experimental de Ituporanga (Epagri/EEltu), SC, in 2019 and 2020 with the hybrid Kybria F1. The experimental design was a randomized block with six replications, with treatments: $80,90,100,110$ and $120 \%$ of the fertilizer quantities recommended by research, following the distribution table indicated by the industry for each stage of development. There was a significant effect of the different concentrations of nutrient solutions evaluated on the yield of cucumber. The treatments that provided the highest productive performance, using the management indicated by the industry, were $90 \%$ of the standard solution and $100 \%$ standard solution.

Index terms: Cucumis sativus; fertigation; phenological phases.

\section{Introdução}

O estado de Santa Catarina é o maior produtor nacional de pepino para conserva, produzindo anualmente 15 mil toneladas, equivalendo a cerca de $25 \%$ da produção nacional. Uma boa parte da produção está concentrada na região do Vale do Itajaí (FÓRUM BRASILEIRO DE PRODUÇÃO DE PEPINO PARA CONSERVA, 2019).

A produtividade dessa hortaliça pode alcançar até $80 \mathrm{t} \mathrm{ha}^{-1}$ em lavouras cultivadas em campo aberto (MARTINS, 2004; REBELO et al., 2011). No entanto, quando cultivado em ambiente protegido, tipo estufa, estima-se que a produtividade obtida em abrigo tenha potencial para superar a produtividade a campo em até $168,5 \%$, dependendo da época de cultivo (VIEIRA NETO et al., 2018). Apesar deste benefício, é necessário agregar outras práticas culturais a fim de potencializar a rentabilidade e reduzir custos de produção de pepineiro cultivado sob abrigo. Dentre elas, destaca-se a utilização de solução nutritiva específica para a cultura cultivada em substrato fertirrigado, levando-se em consideração as diferentes fases fenológicas, taxas de crescimento e absorção de nutrientes.

Andriolo \& Peil (2016) apresentam uma recomendação de solução nutritiva para cultivo de pepineiro em substrato, mas não há uma especificação quanto ao tipo de pepino, salada, japonês ou conserva. No entanto, Espínola et al. (2001) avaliaram o efeito de três níveis múltiplos de uma dose padrão de nutrientes sobre o acúmulo e a distribuição da matéria seca da planta de pepino tipo conserva, híbrido Crispina, cultivado em substrato sob abrigo. Estes autores observaram efeito significativo sobre o acúmulo de matéria seca dos órgãos da parte aérea da planta. Entretanto, relatam que o efeito das doses sobre o crescimento da planta e dos frutos mostrou tendência linear, sem indicar saturação, sugerindo a necessidade de realização de novos experimentos com níveis mais elevados de fertilizantes para determinar o rendimento potencial de frutos do híbrido testado.

$O$ cultivo de pepineiro em substrato vem crescendo na região do Alto Vale do Itajaí, como alternativa ao cultivo tradicional realizado no solo. Isso ocor-

Recebido em 23/6/2020. Aceito para publicação em 23/12/2020.

${ }^{1}$ Engenheiro-agrônomo, Dr., Epagri/Estação Experimental de Ituporanga, C.P. 121, 88400-000 Ituporanga, SC, fone: (47) 3533-8844, e-mail: joaoneto@epagri.sc.gov.br.

2 Engenheiro-agrônomo, Dr., Epagri/Estação Experimental de Itajaí, C.P. 277, 88301-970 Itajaí, SC, fone: (48) 3233-5244, e-mail: rafael.epagri@gmail.com.

${ }^{3}$ Químico, MSc., Epagri/Estação Experimental de Ituporanga, C.P. 121, 88400-000 Ituporanga, SC, fone: (47) 3533-8844, e-mail: cmora@epagri.sc.gov.br. 
re devido à salinização e à incidência de doenças no solo, em consequência do manejo incorreto desse sistema de produção, sem práticas de conservação do solo e pelo uso incorreto dos fertilizantes. Normalmente, os substratos apresentam quantidades de nutrientes insuficientes para o desenvolvimento das plantas, sendo necessário o fornecimento de nutrientes via fertirrigação para atender suas necessidades nutricionais. O presente trabalho foi realizado com objetivo de avaliar concentrações da solução nutritiva sobre a produtividade e a qualidade de frutos de pepineiro para conserva sob ambiente protegido.

\section{Material e métodos}

Dois experimentos foram conduzidos na Epagri, Estação Experimental de Ituporanga, SC $\left(27^{\circ} 38^{\prime}\right.$ de LS e $49^{\circ} 60^{\prime}$ de LO e altitude de $475 \mathrm{~m}$, clima tipo Cfa, segundo a classificação de Köeppen) no segundo semestre de 2019 e primeiro de 2020.

Os estudos foram instalados em estufa com estrutura de aço galvanizado com pé direito de $4,00 \mathrm{~m}$, teto em arco e abertura zenital. $O$ teto era coberto com plástico agrícola difusor de luz ANTI-UV de 150 micras. As cortinas laterais somente foram fechadas na ocorrência de ventos fortes.

O delineamento experimental utilizado foi o de blocos casualizados, com cinco tratamentos e seis repetições, sendo os tratamentos: $80,90,100,110$ e $120 \%$ das quantidades de fertilizantes recomendadas por Andriolo \& Peil (2016) para pepineiros em substrato. A solução padrão $100 \%$ (em $\mathrm{g} \mathrm{L}^{-1}$ ) foi composta das seguintes concentrações de fertilizantes: nitrato de cálcio $(0,865), \operatorname{MKP}(0,204)$, sulfato de magnésio $(0,370)$, nitrato de potássio $(0,425)$, acrescido de ConMicros Standard ${ }^{\circ}$ (composição em \%): B $(1,82)$, Cu EDTA $(1,82)$, Fe $\operatorname{EDTA}(7,26), \operatorname{Mn} \operatorname{EDTA}(1,82)$, Mo $(0,36)$, Ni $(0,335)$, Zn EDTA $(0,73)$, na dose de $0,03 \mathrm{~g} \mathrm{~L}^{-1}$.

Foram utilizados vasos de polietileno com volume de 12 litros cada. As parcelas experimentais foram compostas por quatro plantas do híbrido Kybria F1, uma planta por vaso, sendo os dois vasos centrais considerados como parcela útil.

Para aplicação dos tratamentos foram consideradas cinco fases fenológicas, adaptado de Yara (2018), bem como as proporções de fertilizantes recomendadas para cada fase, conforme descritos na Tabela 1.

As soluções, com as quantidades de fertilizantes para cada tratamento, foram aplicadas diariamente nos vasos usando becker graduado nas quantidades: fase inicial $\left(0,5 \mathrm{~L} \mathrm{planta}^{-1}\right)$, fase vegetativa $1\left(1,0 \mathrm{~L} \mathrm{planta}^{-1}\right)$, fase vegetativa $2\left(1,5 \mathrm{~L}\right.$ planta $\left.^{-1}\right)$, fase vegetativa $3(2,0 \mathrm{~L}$ planta $\left.^{-1}\right)$, fase floração/produção $(2,5 \mathrm{~L}$ planta $\left.^{-1}\right)$. Todas as irrigações foram feitas apenas com soluções nutritivas, utilizando-se água coletada da chuva.

As mudas foram produzidas em bandejas de isopor com 72 células e transplantadas para os vasos nos dias 2 de outubro de 2019 e 13 de janeiro 2020, no estádio de duas folhas definitivas. Os vasos foram arranjados sobre plataforma de madeira com $2,5 \mathrm{~cm}$ de espessura, $25 \mathrm{~cm}$ de largura a $15 \mathrm{~cm}$ de altura do chão, para evitar contato direto com o chão do abrigo, no espaçamento de $0,4 \mathrm{~m} \times 1,0 \mathrm{~m}$. As plantas foram conduzidas verticalmente, com ajuda de fitiIhos plásticos. Foi utilizado o substrato comercial (MaxFértil ${ }^{\circ}$ ) para a produção de mudas e para o cultivo das plantas nos vasos, nos dois anos de avaliação, sendo utilizado substrato novo em cada ensaio. Este substrato possui abundância na região de estudo, composto por uma mistura de casca de pínus compostada e vermiculita.

Segundo análise feita pelo Laboratório de Análise de Substratos para Plantas da Faculdade de Agronomia do Departamento de Horticultura e Silvicultura da UFRGS, o substrato apresenta as seguintes características químicas, conforme extrato aquoso 1:5 (v/v): $\mathrm{pH}$ $\left(\mathrm{H}_{2} \mathrm{O}\right)=5,56 ; \mathrm{CE}=0,55 \mathrm{mS} \mathrm{cm}^{-1}$; densidade seca $=337,5 \mathrm{~kg} \mathrm{~m}^{-3} ;$ umidade atual $=$ $43,7 \%$; porosidade total $=79,6 \%$; espaço de aeração = 26,0\%; água facilmente disponível = 16,4\%; água tamponante = 2,8\%; água remanescente = 34,2\%; e capacidades de retenção de água a 10 $\mathrm{cm}=53,5 \%, 50 \mathrm{~cm}=37,1 \%$ e $100 \mathrm{~cm}=$ $30,2 \%$.

Durante o experimento foram medidos semanalmente, por meio de peagâmetro e condutivímetro, o pH e a condutividade elétrica das soluções nutritivas de cada tratamento (solução aplicada e drenada) para verificar a adequação desses parâmetros em cada fase da cultura. O volume de solução nutritiva foi aplicado para possibilitar uma fração de drenagem da ordem de 20 a 30\%.

Amostras foliares foram retiradas das plantas no início do florescimento para quantificar valores de nutrientes, conforme preconizado pela Comissão de Química e Fertilidade do Solo (CQFSRS/SC, 2004). As análises de tecido das folhas foram realizadas conforme Tedesco et al. (1995).

Em 2019 a colheita de pepino teve início no dia 13/11/2019 e estendeu-

Tabela 1. Fase fenológica, intervalo de cada fase, porcentagem do total da mistura de fertilizantes aplicada em cada fase (PMF) e quantidade de solução da mistura de fertilizantes fornecida por planta a cada dia em cada tratamento por fase fenológica Table 1. Phenological phase, interval of each phase, percentage of total fertilizer mixture applied in each phase (PFM) and amount of fertilizer mixture solution supplied per plant each day in each treatment per phenological phase

\begin{tabular}{lcccccccc}
\hline & & & \multicolumn{7}{c}{ Mistura de fertilizantes $\left(\mathrm{g} \mathrm{L}^{-1}\right)$} \\
\cline { 5 - 8 } Fase fenológica & $\begin{array}{c}\text { Intervalo } \\
\text { (Dias) }\end{array}$ & $\begin{array}{c}\text { PMF } \\
\text { (\%) }\end{array}$ & $\mathbf{8 0 \%}$ & $\mathbf{9 0 \%}$ & $\mathbf{1 0 0 \%}$ & $\mathbf{1 1 0 \%}$ & $\mathbf{1 2 0 \%}$ \\
\hline Fase inicial & 1 a 10 & 13 & 0,202 & 0,228 & 0,253 & 0,278 & 0,304 \\
\hline Vegetativa 1 & 11 a 20 & 27 & 0,405 & 0,456 & 0,507 & 0,557 & 0,608 \\
\hline Vegetativa 2 & 21 a 30 & 76 & 1,160 & 1,305 & 1,450 & 1,595 & 1,740 \\
\hline Vegetativa 3 & 31 a 40 & 80 & 1,216 & 1,369 & 1,521 & 1,673 & 1,825 \\
\hline $\begin{array}{l}\text { Floração/ } \\
\text { produção }\end{array}$ & $>41$ & 100 & 1,525 & 1,715 & 1,906 & 2,097 & 2,287 \\
\hline
\end{tabular}


se até 06/01/2020, totalizando 21 coIheitas. Em 2020 foram realizadas oito colheitas no período de 02/03/2020 a $18 / 03 / 2020$. As colheitas foram realizadas em média a cada dois dias, quando os frutos apresentavam entre 4 a $9 \mathrm{~cm}$ de comprimento, padrão exigido pelas agroindústrias (REBELO et al., 2011).

Foram avaliadas as seguintes variáveis: o número de frutos comerciais por planta, a porcentagem de frutos comerciais e não comerciais (frutos com mais de $9 \mathrm{~cm}$ de comprimento, defeituosos e danificados por pragas), a massa fresca de frutos comerciais (g) e a massa fresca de frutos comerciais por planta (kg planta-1). Os dados dessas variáveis acumulados até a última colheita foram submetidos à análise de variância pelo teste $\mathrm{F}$ e as médias comparadas pelo teste de Scott-Knott, a 5\% de probabilidade, utilizando-se o software livre " $R$ ". Os valores médios de macro e micronutrientes foliares encontrados nas amostras foram comparados aos valores de nutrientes considerados adequados para o pepineiro segundo a CQFS-RS/SC (2004) pelo teste $t$ de Student a 5\% de probabilidade.

\section{Resultados e discussão}

Houve efeito significativo dos tratamentos sobre a massa fresca de frutos comerciais por planta (MFFP) apenas no experimento de 2019 (Tabela 2). Os melhores resultados foram obtidos com os tratamentos $90 \%$ e $100 \%$, com médias de 2,69 e 2,87 kg planta-1, respectivamente. Em 2020, foram observados valores inferiores aos obtidos em 2019 para a MFFP, com valores variando entre 0,55 a $0,65 \mathrm{~kg}$ planta-1. Os baixos valores para MFFP observados em 2020 foram consequência de um ataque severo de pulgões, associados a sintomas de viroses. Este ataque reduziu o ciclo de cultivo e o número de colheitas. Em 2019 foram realizadas 21 colheitas, enquanto que em 2020 apenas oito.

A radiação solar pode ter sido o principal fator climático a ter contribuído para a maior incidência de pulgões nas plantas em 2020. Durante o período de colheita de 2020, com duração de 16 dias, a soma de radiação média diária foi de $3.724 \mathrm{~W} / \mathrm{m}^{2}$, valor $10,81 \%$ inferior

Tabela 2. Médias de massa fresca de frutos comerciais por planta (MFFP), número de frutos comerciais por planta (NFP), massa fresca de frutos comerciais (MFF), porcentagem de frutos comerciais (\%FC) e porcentagem de frutos não comerciais (\%FNC), para pepino em conserva nos diferentes tratamentos, obtidas no final dos experimentos ${ }^{1}$. Ituporanga, SC, Epagri, 2019 e 2020

Table 2. Means of fresh weight of commercial fruits per plant (MFFP), number of commercial fruits per plant (NFP), fresh weight of commercial fruits (MFF), percentage of commercial fruits (\% FC) and percentage of non-commercial fruits (\% FNC), for pickling cucumber in the different treatments, obtained at the end of the experiments. Ituporanga, SC, Epagri, 2019 and 2020

\begin{tabular}{|c|c|c|c|c|c|c|}
\hline & 2019 & 2020 & 2019 & 2020 & 2019 & 2020 \\
\hline Tratamentos & \multicolumn{2}{|c|}{ MFFP (kg planta-1) } & \multicolumn{2}{|c|}{ NFP } & \multicolumn{2}{|c|}{ MFF (g) } \\
\hline $80 \%$ & $2,59 \mathrm{~b}$ & 0,61 NS & $154,4 \mathrm{a}$ & $34,0 \mathrm{a}$ & $16,8^{\mathrm{NS}}$ & $17,9 \mathrm{~b}$ \\
\hline $90 \%$ & $2,69 a$ & 0,59 & 161,6 a & 32,7 a & 16,7 & $18,3 \mathrm{~b}$ \\
\hline $100 \%$ & $2,87 a$ & 0,65 & $171,4 \mathrm{a}$ & 33,7 a & 16,7 & 19,4 a \\
\hline $110 \%$ & $2,40 \mathrm{~b}$ & 0,55 & $142,1 \mathrm{~b}$ & $28,1 b$ & 17,1 & 19,4 a \\
\hline $120 \%$ & $2,40 \mathrm{~b}$ & 0,55 & $139,4 \mathrm{~b}$ & $27,9 \mathrm{~b}$ & 17,2 & $19,8 \mathrm{a}$ \\
\hline CV\% & 10,62 & 14,52 & 10,39 & 16,06 & 8,15 & 5,67 \\
\hline Ano/ & 2019 & & 2020 & 2019 & & 2020 \\
\hline Tratamentos & \multicolumn{3}{|c|}{$\% F C$} & \multicolumn{3}{|c|}{$\%$ FNC } \\
\hline $80 \%$ & $97,7^{\mathrm{NS}}$ & & 98,9 NS & 2,3 NS & & $1,02^{\mathrm{NS}}$ \\
\hline $90 \%$ & 98,0 & & 99,7 & 2,0 & & 0,26 \\
\hline $100 \%$ & 97,2 & & 98,7 & 2,8 & & 1,23 \\
\hline $110 \%$ & 98,1 & & 96,8 & 1,9 & & 3,13 \\
\hline $120 \%$ & 97,7 & & 96,9 & 2,3 & & 3,03 \\
\hline CV\% & 0,96 & & 2,50 & 38,01 & & 118,35 \\
\hline
\end{tabular}

${ }^{1}$ Médias não seguidas da mesma letra minúscula na vertical, dentro da mesma variável, diferem entre si pelo teste de Scott-Knott a 5\% de probabilidade. NS: Não significativo pelo teste F a 5\%.

ao observado para o mesmo período de colheita em 2019. Neste mesmo período as médias de temperatura média e umidade relativa do ar apresentaram pouca variação entre os anos avaliados, respectivamente, $2019\left(20,11^{\circ} \mathrm{C}\right.$; $73,70 \%)$ e $2020\left(21,82^{\circ} \mathrm{C} ; 76,62 \%\right)$.

Estudo realizado por Vieira Neto et al. (2019) em condição experimental semelhante, na mesma localidade, alcançou rendimento de 1,83kg de MFFP para o híbrido Kybria. Este resultado equivale a $34,2 \%$ a menos na produção em comparação à média resultante dos dois melhores tratamentos do presente trabalho, $90 \%$ e $100 \%$ (2,78kg planta $\left.{ }^{-1}\right)$. Por outro lado, Espínola et al. (2001) obtiveram produtividade próxima a $31 \mathrm{t}$ ha ${ }^{-1}$, equivalente a $1,55 \mathrm{~kg}_{\text {planta }}{ }^{-1}$, para pepino tipo conserva, híbrido Crispina, cultivado em substrato sob abrigo em
Santa Maria, estado do Rio Grande do Sul.

O número de frutos comerciais por planta (NFP) foi afetada pelos tratamentos nos dois anos de avaliação (Tabela 2). Em 2019, observou-se maior número de frutos comerciais por planta para $80 \%(154,4), 90 \%(161,6)$ e $100 \%(171,4)$ e, em 2020 , para $80 \%(34,0), 90 \%(32,7)$ e $100 \%(33,7)$. Do mesmo modo que ocorrido com MFFP em 2020, o NFP foi afetado pela incidência de pulgões nas plantas de pepineiro naquele ano.

Quanto à massa fresca de frutos comerciais (MFF), em 2019, não houve diferença entre os diferentes tratamentos, variando entre 16,7 a 17,2g (Tabela 2). Em 2020, houve efeito de tratamentos, sendo que os maiores valores para MFF foram de 19,4, 19,4, e 19,8g obtidos com os tratamentos $100 \%, 110 \%$ 
e $120 \%$, respectivamente. Estes resultados são compatíveis aos valores normalmente encontrados em trabalhos feitos com pepino tipo conserva, devido à existência de um padrão de colheita previamente estabelecido pela agroindústria para frutos destinados ao envasamento. Ou seja, os resultados encontrados por Vieira Neto et al. (2018) e Vieira Neto et al. (2019) situam entre 18,53 a $21,52 \mathrm{~g}$.

As porcentagens de frutos comerciais e de frutos não comerciais não foram influenciadas pelos tratamentos nos dois anos de avaliação (Tabela 2). $\mathrm{O}$ rendimento médio de frutos comercializáveis dos cinco tratamentos foi de $97,7 \%$ e 98,2\% para 2019 e 2020, respectivamente. Resultado bem próximo a este foi encontrado por Vieira Neto et al. (2019), com 98,3\% dos frutos do cultivar Kybria com padrão comercial.

A boa qualidade dos frutos, refletida na alta porcentagem de frutos comerciais, sugere um bom equilíbrio entre os nutrientes nas soluções nutritivas utilizadas, e que a quantidade de nutrientes associada ao manejo nutricional adequado são os fatores mais determinantes nas elevações de produtividade do pepineiro em substrato.

Na Tabela 3 é possível verificar que os níveis de nutrientes foliares para macronutrientes, de maneira geral, não foram influenciados pelos tratamentos. Embora apenas P esteja dentro dos valores de referência definidos pela Comissão de Química e Fertilidade do Solo (CQFS-RS/SC, 2004), os demais nutrientes, mesmo estando abaixo ( $\mathrm{N}$, $\mathrm{K}, \mathrm{S}$ ) e acima ( $\mathrm{Ca}, \mathrm{Mg}$ ), estão próximos aos valores dos limites de intervalos de normalidade para a cultura.

Quanto aos micronutrientes (Tabela 4), os níveis foliares amostrais apresentaram-se adequados para $\mathrm{B}$ e $\mathrm{Cu}$, para todos os tratamentos, e para Fe (80\%), para $\mathrm{Mn}((80 \%, 90 \%, 100 \%$ e $120 \%)$ e $\mathrm{Zn}$ (100\% e $120 \%)$. Assim como observado para macronutrientes, esse resultado sugere uma falta de relação entre as concentrações de nutrientes dos diferentes tratamentos com os níveis dos elementos nas folhas. No entanto, Diniz et al. (2015) verificaram que a aplicação de diferentes proporções de nutrientes na solução nutritiva exerceu efeitos significativos sobre os teores foliares de N,
Tabela 3. Valores médios de macronutrientes e semiamplitude do intervalo de confiança para a média, nas folhas de pepineiro em \%. Ituporanga, SC, Epagri, 2019 e 2020 Table 3. Mean values of macronutrients and semiamplitude of the confidence interval for the mean, in cucumber leaves in \%. Ituporanga, SC, Epagri, 2019 and 2020

\begin{tabular}{|c|c|c|c|c|c|c|}
\hline Tratamento & N\% & $\mathrm{P} \%$ & $\mathrm{~K} \%$ & $\mathrm{Ca} \%$ & $\mathrm{Mg} \%$ & $\mathrm{~S} \%$ \\
\hline $80 \%$ & $\begin{array}{l}3,2 \operatorname{lnf} . \\
(0,68)\end{array}$ & $\begin{array}{l}0,9 \mathrm{NA} \\
(0,16)\end{array}$ & $\begin{array}{l}2,5 \text { inf. } \\
(0,46)\end{array}$ & $\begin{array}{l}6,5 \text { sup. } \\
(1,35)\end{array}$ & $\begin{array}{l}2,4 \text { Sup. } \\
(0,33)\end{array}$ & $\begin{array}{l}0,3^{\operatorname{lnf} .} \\
(0,03)\end{array}$ \\
\hline $90 \%$ & $\begin{array}{l}3,0^{\operatorname{lnf} .} \\
(0,56)\end{array}$ & $\begin{array}{l}0,9 \mathrm{NA} \\
(0,14)\end{array}$ & $\begin{array}{l}2,6 \text { Inf. } \\
(0,43)\end{array}$ & $\begin{array}{l}6,7 \text { Sup. } \\
(0,31)\end{array}$ & $\begin{array}{l}2,4 \text { sup. } \\
(0,29)\end{array}$ & $\begin{array}{l}0,2^{\operatorname{lnf} .} \\
(0,06)\end{array}$ \\
\hline $100 \%$ & $\begin{array}{l}2,3^{\text {Inf. }} \\
(1,07)\end{array}$ & $\begin{array}{l}0,9^{N A} \\
(0,22)\end{array}$ & $\begin{array}{l}2,4 \text { inf. } \\
(0,37)\end{array}$ & $\begin{array}{l}7,1 \text { Sup. } \\
(1,28)\end{array}$ & $\begin{array}{l}2,5 \text { Sup. } \\
(0,50)\end{array}$ & $\begin{array}{l}0,2^{\operatorname{lnf} .} \\
(0,03)\end{array}$ \\
\hline $110 \%$ & $\begin{array}{l}3,7^{\operatorname{lnf} .} \\
(0,48)\end{array}$ & $\begin{array}{l}1,0 \mathrm{NA} \\
(0,17)\end{array}$ & $\begin{array}{l}2,7 \text { Inf. } \\
(0,75)\end{array}$ & $\begin{array}{l}7,3 \text { sup. } \\
(0,96)\end{array}$ & $\begin{array}{l}2,4 \text { Sup. } \\
(0,22)\end{array}$ & $\begin{array}{l}0,2^{\operatorname{lnf} .} \\
(0,08)\end{array}$ \\
\hline $120 \%$ & $\begin{array}{l}3,6^{\operatorname{lnf} .} \\
(0,58)\end{array}$ & $\begin{array}{l}0,9 \mathrm{NA} \\
(0,13)\end{array}$ & $\begin{array}{l}2,4 \text { inf. } \\
(0,49)\end{array}$ & $\begin{array}{l}7,1 \text { sup. } \\
(0,83)\end{array}$ & $\begin{array}{l}2,5 \text { sup. } \\
(0,32)\end{array}$ & $\begin{array}{l}0,2^{\operatorname{lnf} .} \\
(0,06)\end{array}$ \\
\hline Referência ${ }^{1}$ & $4,5-6,0$ & $0,3-1,2$ & $3,5-5,0$ & $1,5-3,5$ & $0,3-1,0$ & $0,4-0,7$ \\
\hline
\end{tabular}

${ }^{1}$ Faixa de referência com base na Comissão de Química e Fertilidade do Solo (CQFS-RS/SC, 2004). Inferior (Inf.), nível adequado (NA) e superior (Sup.) aos valores de referência, significativos pelo teste $t$ de Student a 5\%.

P, K, Ca e Mg das folhas de plantas do híbrido de pepineiro Magnum F1, grupo caipira, cultivadas em substrato de fibra de coco. Esses autores utilizaram soluções nutritivas nas concentrações de $12,5 \%, 17 \%, 25 \%$, $50 \%$ e $100 \%$ da solução nutritiva sugerida por Furlani et al. (1999).

Os níveis de $\mathrm{pH}$ oscilaram entre 5,70 a 6,45 e entre 5,60 a 6,50 , respectivamente, para a solução nutritiva aplicada e drenada nos distintos tratamentos e fases (Tabela 5). Os níveis observados de $\mathrm{pH}$, nos experimentos, estão dentro dos parâmetros considerados ideais para solução nutritiva indicada por An-»

Tabela 4. Valores médios de micronutrientes e semi-amplitude do intervalo de confiança para a média, nas folhas de pepineiro em mg kg-1. Ituporanga, SC, Epagri, 2019 e 2020 Table 4. Means values of micronutrients and semi-amplitude of the confidence interval for the mean, in cucumber leaves in mg/kg. Ituporanga, SC, Epagri, 2019 and 2020

Tratamento $\quad \mathrm{B}\left(\mathrm{mg} \mathrm{kg}^{-1}\right) \quad \mathrm{Cu}\left(\mathrm{mg} \mathrm{kg}^{-1}\right) \quad \mathrm{Fe}\left(\mathrm{mg} \mathrm{kg}^{-1}\right) \quad \mathrm{Mn}\left(\mathrm{mg} \mathrm{kg}^{-1}\right) \mathrm{Zn}\left(\mathrm{mg} \mathrm{kg}^{-1}\right)$

\begin{tabular}{|c|c|c|c|c|c|}
\hline $80 \%$ & $\begin{array}{c}66,5^{\mathrm{NA}} \\
(7,4)\end{array}$ & $\begin{array}{l}12,3^{\mathrm{NA}} \\
(2,80)\end{array}$ & $\begin{array}{l}408,1^{N A} \\
(116,04)\end{array}$ & $\begin{array}{l}252,0^{N A} \\
(48,73)\end{array}$ & $\begin{array}{c}143,6 \text { sup. } \\
(15,81)\end{array}$ \\
\hline $90 \%$ & $\begin{array}{c}78,7^{\mathrm{NA}} \\
(8,97)\end{array}$ & $\begin{array}{c}12,3^{\mathrm{NA}} \\
(2,53)\end{array}$ & $\begin{array}{c}528,7 \text { Sup. } \\
(42,54)\end{array}$ & $\begin{array}{c}267,4^{N A} \\
(48,24)\end{array}$ & $\begin{array}{c}129,4 \text { sup. } \\
(22,84)\end{array}$ \\
\hline $100 \%$ & $\begin{array}{l}75,6^{\mathrm{NA}} \\
(12,31)\end{array}$ & $\begin{array}{c}11,8^{\mathrm{NA}} \\
(2,58)\end{array}$ & $\begin{array}{l}737,6 \text { Sup. } \\
(216,68)\end{array}$ & $\begin{array}{c}291,9 \text { NA } \\
(59,68)\end{array}$ & $\begin{array}{c}116,0^{\mathrm{NA}} \\
(27,87)\end{array}$ \\
\hline $110 \%$ & $\begin{array}{l}70,0 \text { NA } \\
(12,22)\end{array}$ & $\begin{array}{c}14,9 \mathrm{NA} \\
(3,76)\end{array}$ & $\begin{array}{l}710,0 \text { Sup. } \\
(175,30)\end{array}$ & $\begin{array}{c}337,4 \text { Sup. } \\
(63,69)\end{array}$ & $\begin{array}{c}133,9 \text { sup. } \\
(30,21)\end{array}$ \\
\hline $120 \%$ & $\begin{array}{l}83,0 \text { NA } \\
(12,52)\end{array}$ & $\begin{array}{l}14,4^{\mathrm{NA}} \\
(3,68)\end{array}$ & $\begin{array}{l}644,6 \text { Sup. } \\
(138,31)\end{array}$ & $\begin{array}{c}328,7^{\mathrm{NA}} \\
(82,94)\end{array}$ & $\begin{array}{c}116,2^{\mathrm{NA}} \\
(21,03)\end{array}$ \\
\hline Referência ${ }^{1}$ & $50-120$ & $7-20$ & $50-300$ & $50-250$ & $25-100$ \\
\hline
\end{tabular}


driolo \& Peil, (2016), que é de 5,5 e 6,5. Quanto à condutividade elétrica (CE) (Tabela 5), para os tratamentos $110 \%$ e $120 \%$, com maiores concentrações e fertilizantes na solução aplicada, os valores oscilaram, respectivamente, entre 0,94 a $2,37 \mathrm{mS} \mathrm{cm}^{-1}$ e 0,98 a $2,67 \mathrm{mS} \mathrm{cm}^{-1} \mathrm{da}$ fase inicial à fase de produção/floração. Os maiores valores de $\mathrm{CE}$, observados principalmente na fase de produção/ floração, acima do parâmetro de referência indicado para solução nutritiva em cultivo de pepineiro, ou seja, $1,8 \mathrm{mS}$ $\mathrm{cm}^{-1}$ (ANDRIOLO \& PEIL, 2016), podem ter contribuído com o menor número de frutos por planta, refletindo numa menor produção. Pois, conforme observado por Diniz et al. (2015), o pepino absorve e utiliza grande quantidade de nutrientes, mas é muito sensível ao excesso de fertilizantes na solução do solo. Para os tratamentos $90 \%\left(1,95 \mathrm{mS} \mathrm{cm}^{-1}\right)$ e $100 \%\left(2,12 \mathrm{mS} \mathrm{cm}^{-1}\right)$ os valores da CE situaram-se mais próximos do valor de referência, com ganhos de produção.

A estratégia de fornecer as soluções nutritivas em diferentes concentrações de fertilizantes, considerando as cin- co fases fenológicas proposta por Yara (2018), pode ter contribuído para a manutenção dos valores de CE um pouco mais próximos aos valores de referência propostos pelo referido autor, para cada fase de desenvolvimento das plantas de pepineiro, a saber: fase $1\left(0,33 \mathrm{mS} \mathrm{cm}^{-1}\right)$, vegetativa $1\left(0,64 \mathrm{mS} \mathrm{cm}^{-1}\right)$, vegetativa 2 $\left(1,03 \mathrm{mS} \mathrm{cm}^{-1}\right)$, vegetativa $3(0,88 \mathrm{mS} \mathrm{cm}$ $\left.{ }^{1}\right)$ e floração/produção $\left(0,88 \mathrm{mS} \mathrm{cm}^{-1}\right)$.

Desta forma, as soluções nutritivas representadas pelos tratamentos $90 \%$ e $100 \%$, utilizando o manejo adotado no presente trabalho para a elaboração das soluções nutritivas ao longo do ciclo de cultivo, são indicadas no manejo do pepineiro fertirrigado por terem proporcionado maior produtividade.

\section{Conclusões}

O melhor desempenho de produção de pepineiro para conserva, em semihidroponia, foi obtido por soluções nutritivas com $90 \%$ e $100 \%$ das quantidades de fertilizantes recomendadas pela pesquisa.
O manejo da solução nutritiva indicado pela indústria, considerando cinco fases fenológicas, bem como as proporções de fertilizantes recomendadas para cada fase, pode servir como referência para produção de pepino para conserva em cultivo semi-hidropônico.

\section{Agradecimentos}

Aos colaboradores técnico agrícola David Junior Rodrigues e assistente de campo Marcelo Andrade, da Epagri, Estação Experimental de Ituporanga, SC.

\section{Referências}

ANDRIOLO, J.L.; PEIL, R.M.N. Sistemas especiais de produção. In: Leandro Souza da Silva; Luciano Colpo Gatiboni. (Org.). Manual de Adubação e Calagem para os estados do Rio Grande do Sul e Santa Catarina. 11ed. Porto Alegre: Comissão de Química e Fertilidade do Solo- RS/SC, 2016, v., p. 287-293.

CQFS-RS/SC. Manual de calagem e adubação para os estados do Rio Grande do Sul

Tabela 5. Médias de duas safras 2019/20 do potencial hidrogeniônico (pH) e condutividade elétrica (CE, $\mathrm{mS} \mathrm{cm}^{-1}$ ) da solução nutritiva aplicada e da solução drenada do substrato. Ituporanga, SC, Epagri, 2019 e 2020

Table 5. Means of two harvests 2019/20 of hydrogen potential (pH) and electrical conductivity (EC, $m S \mathrm{~cm}-1)$ of the applied nutrient solution and the solution drained from the substrate. Ituporanga, SC, Epagri, 2019 and 2020

\begin{tabular}{|c|c|c|c|c|c|c|c|c|c|c|}
\hline \multirow[b]{3}{*}{ Fase fenológica } & \multicolumn{10}{|c|}{ Solução aplicada } \\
\hline & \multicolumn{2}{|c|}{$80 \%$} & \multicolumn{2}{|c|}{$90 \%$} & \multicolumn{2}{|c|}{$100 \%$} & \multicolumn{2}{|c|}{$110 \%$} & \multicolumn{2}{|c|}{$120 \%$} \\
\hline & $\mathrm{pH}$ & $\mathrm{CE}$ & $\mathrm{pH}$ & CE & $\mathrm{pH}$ & $\mathrm{CE}$ & $\mathrm{pH}$ & $\mathrm{CE}$ & $\mathrm{pH}$ & $\mathrm{CE}$ \\
\hline Fase 1 (inicial) & 6,40 & 0,64 & 6,43 & 0,74 & 6,37 & 0,80 & 6,33 & 0,94 & 6,20 & 0,98 \\
\hline Vegetativa 1 & 6,45 & 0,69 & 6,40 & 0,76 & 6,30 & 0,80 & 6,30 & 0,98 & 6,25 & 1,01 \\
\hline Vegetativa 2 & 6,28 & 0,73 & 6,43 & 0,78 & 6,35 & 0,83 & 6,25 & 1,03 & 6,20 & 1,03 \\
\hline Vegetativa 3 & 5,90 & 1,21 & 5,90 & 1,39 & 5,73 & 1,95 & 5,73 & 2,32 & 5,70 & 2,50 \\
\hline \multirow[t]{2}{*}{ Floração/produção } & 6,08 & 1,21 & 5,95 & 1,95 & 5,96 & 2,12 & 5,85 & 2,37 & 5,81 & 2,67 \\
\hline & \multicolumn{10}{|c|}{ Solução drenada } \\
\hline Fase fenológica & $\mathrm{pH}$ & CE & $\mathrm{pH}$ & CE & pH & CE & $\mathrm{pH}$ & CE & $\mathrm{pH}$ & CE \\
\hline Fase 1 (inicial) & 6,37 & 0,43 & 6,27 & 0,66 & 6,03 & 0,68 & 5,83 & 0,89 & 5,70 & 0,92 \\
\hline Vegetativa 1 & 6,50 & 0,47 & 5,90 & 0,68 & 5,95 & 0,70 & 5,75 & 0,92 & 5,60 & 0,95 \\
\hline Vegetativa 2 & 6,20 & 0,59 & 5,90 & 0,69 & 6,20 & 0,70 & 5,70 & 0,92 & 5,60 & 0,91 \\
\hline Vegetativa 3 & 6,10 & 0,66 & 6,10 & 0,84 & 5,77 & 1,50 & 5,80 & 2,07 & 5,63 & 2,23 \\
\hline Floração/produção & 6,31 & 0,72 & 5,91 & 0,91 & 5,81 & 1,80 & 5,82 & 2,18 & 5,60 & 2,46 \\
\hline
\end{tabular}


e de Santa Catarina. 10.ed. Porto Alegre, RS: SBCS/Núcleo Regional Sul; Comissão de Química e Fertilidade do Solo-RS/SC, 2004. 400p.

DINIZ, A. A.; DIAS, N. da S.; SOUZA, F. I. de S.; SOUZA, A. C. M.; MESQUITA, F. de O.; SOUZA, F. I. de. Efeito da solução nutritiva sob o crescimento e composição mineral em pepino cultivado em substrato de fibra de coco. Semina: Ciências Agrárias (Online), v. 36, p. 3043-3054, 2015. Disponível em: https://www.researchgate.net/publication/283686114_Efeito_da_solucao_nutritiva_sob_o_crescimento_e_composicao_mineral_em_pepino_cultivado_em_substrato_de_fibra_de_coco. Acesso em: 25 maio 2020.

ESPÍNOLA, H.N.R.; ANDRIOLO, J.L.; BARTZ, H.R. Acúmulo e repartição da matéria seca da planta de pepino tipo conserva sob três doses de nutrientes minerais. Ciência Rural, Santa Maria, RS, v. 31, n.3, p. 387-392, 2001. Disponível em: https://www.scielo.br/ pdf/cr/v31n3/a04v31n3.pdf. Acesso em: 11 maio 2020.
FÓRUM BRASILEIRO DE PRODUÇÃO DE PEPINO PARA CONSERVA, 2. 2019, Indaial. Produção sustentável com alta tecnologia. Disponível em: http://forumbrasilpepinoconserva.com.br/como-proteger-a-producaode-pepinos-de-pragas-ou-doencas/. Acesso em: 21 maio 2020.

FURLANI, P. R.; BOLONHEZI, D.; SILVEIRA, L. C. P.; FAQUIN, V. Nutrição mineral de hortaliças, preparo e manejo de soluções nutritivas. Informe Agropecuário: Cultivo protegido de hortaliças em solo e hidroponia, v. 20, n. 200/201, p. 90-98, 1999.

MARTINS, C.N. Pepino: produção triplicada. Revista Cultivar Hortaliças e Frutas, Pelotas, 2004. Disponível em: http://www.grupocultivar.com.br/arquivos/hf24_producao.pdf. Acesso em: 19 abr. 2020.

REBELO, J.A.; SCHALLENBERGER, E.; CANTÚ, RR. Cultivo do pepineiro para picles no Vale do Rio Itajaí e Litoral Catarinense. Florianópolis: Epagri, 2011. 55p. (Boletim Técnico, 154).
TEDESCO, M.J.; GIANELLO, C.; BISSANI, C.A.; BOHNEN, H. Análise de solo, plantas e outros materiais. 2.ed. Porto Alegre: UFRGS, 1995. (Boletim Técnico, 5).

VIEIRA NETO, J.; GONÇALVES, P.A. de S.; MENEZES JÚNIOR, F.O.G. de. Produtividade de cultivares de pepino para conserva em diferentes épocas de plantio sob ambiente protegido. Revista Thema, v. 15, p. 93-101, 2018. Disponível em: http://periodicos.ifsul. edu.br/index.php/thema/article/view/809. Acesso em: 05 maio 2020.

VIEIRA NETO, J.; MENEZES JÚNIOR, F.O.G. de; GONÇALVES, P.A. de S. Avaliação da produção de cultivares de pepino para conserva e de mini tomate com e sem aplicação de fertilizante foliar. Revista Thema, v.16, p.855-864, 2019. Disponível em: http://periodicos.ifsul.edu.br/index.php/thema/article/view/1473. Acesso em: 05 maio 2020.

YARA. Yara GranHorti: Programa nutricional para pepino - fetirrigação. [2018]. 40p.
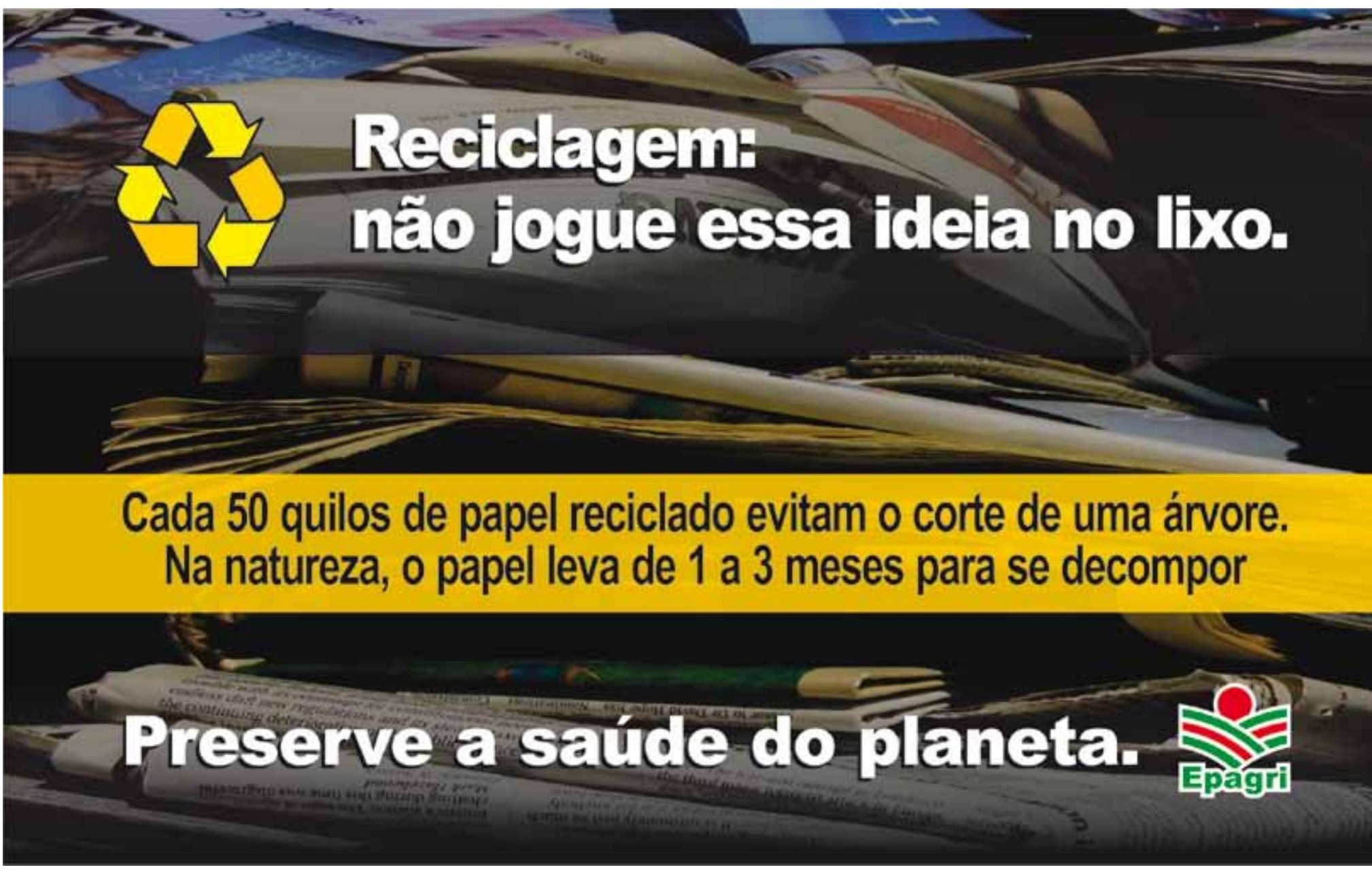